\title{
Experiences of Latent Tuberculosis Infection Treatment for the North Korean Refugees
}

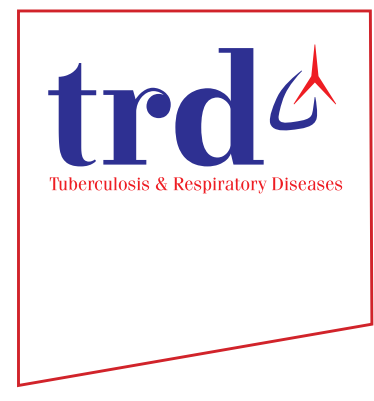

Beong Ki Kim, M.D. ${ }^{1}{ }^{(}$, Hee Jin Kim, M.D. ${ }^{2}$, Ho Jin Kim, M.D. ${ }^{1}$, Jae Hyung Cha, Ph.D. ${ }^{3}$, Jin Beom Lee, M.S. ${ }^{2}$, Jeonghe Jeon, M.Sc. ${ }^{4}$, Chi Young Kim, M.D. ${ }^{1}$, Young Kim, M.D. ${ }^{1}$, Je Hyeong Kim, M.D., Ph.D. ${ }^{1}$, Chol Shin, M.D., Ph.D. ${ }^{1}$ and Seung Heon Lee, M.D., Ph.D. ${ }^{1}$ (i)

${ }^{1}$ Division of Pulmonary, Sleep and Critical Care Medicine, Department of Internal Medicine, Korea University Ansan Hospital, Korea University College of Medicine, Ansan, ${ }^{2}$ Korea Institute of Tuberculosis, Cheongju, ${ }^{3}$ Medical Science Research Center, Korea University Ansan Hospital, Korea University College of Medicine, Ansan, ${ }^{4}$ Settlement Support Center for North Korean Refugees (Hanawon), Hwacheon, Korea

Background: Tuberculosis (TB) is increasing in immigrants. We aimed to investigate the current status of latent tuberculosis infection (LTBI) treatment for North Korean Refugees (NKR) compared to South Koreans Contacts (SKC). Methods: TB close contacts in a closed facility of SKC and NKR who underwent LTBI screening in a settlement support center for NKR were analyzed retrospectively.

Results: Among tuberculin skin test (TST) $\geq 10 \mathrm{~mm}(\mathrm{n}=298)$ reactors, the males accounted for $72.2 \%$ in SKC ( $\mathrm{n}=126)$ and $19.5 \%$ in NKR $(\mathrm{n}=172)(\mathrm{p}<0.01)$. The mean age was higher in South Korea $(42.8 \pm 9.9$ years vs. $35.4 \pm 10.0$ years, $\mathrm{p}<0.01)$. Additionally, the mean TST size was significantly bigger in NKR $(17.39 \pm 3.9 \mathrm{~mm}$ vs. $16.57 \pm 4.2 \mathrm{~mm}, \mathrm{p}=0.03)$. The LTBI treatments were initiated for all screened NKR, and LTBI completion rate was only $68.0 \%$. However, in NKR, LTBI treatment completion rate was significantly increased by shorter $4 \mathrm{R}$ regimen (odds ratio [OR], 9.296; 95\% confidence interval [CI], 4.159-20.774; $\mathrm{p}<0.01$ ) and male (OR, 3.447; 95\% CI, 1.191-9.974; $\mathrm{p}=0.02$ ).

Conclusion: LTBI treatment compliance must be improved in NKR with a shorter regimen. In addition, a larger study regarding a focus on LTBI with easy access to related data for NKR should be conducted.

Keywords: Latent Tuberculosis Infection; Tuberculin Test; Interferon-Gamma Release Tests; Therapeutics; Asian Continental Ancestry Group; Refugees

Address for correspondence: Seung Heon Lee, M.D., Ph.D.

Division of Pulmonary, Sleep and Critical Care Medicine, Department of Internal Medicine, Korea University Ansan Hospital, 123 Jeokgeum-ro, Danwon-gu, Ansan 15355, Korea

Phone: 82-31-412-4973, Fax: 82-31-412-4975

E-mail: lee-sh@korea.ac.kr

Received: Apr. 24, 2019

Revised: May. 9, 2019

Accepted: May. 17, 2019

Published online: May. 31, 2019

(c) It is identical to the Creative Commons Attribution Non-Commercial License (http://creativecommons.org/licenses/by-nc/4.0/).

\section{Introduction}

Tuberculosis (TB) is known to be the leading cause of death among infectious diseases worldwide. However, TB incidence in South Korea (SK) has been continuously decreased to $65.9 / 100,000$ population in $2018^{1}$. Recently, latent TB infection (LTBI) treatment with political commitment accompanied by promotion of human rights has been addressed by World Health Organization $^{2}$ as the END TB strategy to prevent the development of TB cases.

The percentage of immigrant TB in SK is $5.3 \%$ in $2018^{1}$. TB cases in immigrants are increasing every year $^{3-5}$. Moreover, many refugees from North Korea (NK) where TB incidence is as high as 513/100,000 population and drug maintenance system is infirm are emigrating to SK every year ${ }^{6,7}$. Korean Government is screening both TB and LTBI for North Korean 
Refugees (NKR) from the immigration process in National Intelligence Service for security ${ }^{3}$.

For LTBI diagnosis, additional confirmatory QuantiFERONTB Gold In Tube (QFT-GIT) test as a two-step strategy had been used for TB contacts with $10 \leq$ tuberculin skin test (TST) induration $<15 \mathrm{~mm}$ with bacillus Calmette-Guérin (BCG) scar or identified BCG vaccination in $\mathrm{SK}^{8,9}$. For LTBI treatment of immigrants, the adequacy based on TB incidence rate and drug resistance rate of emigration country must be weighed against the benefit of LTBI treatment ${ }^{10}$. In case of vulnerable NKR, drug compliance and inaccessibility to health center owing to political and cultural barrier seem to be crucial ${ }^{11}$ for LTBI control policy. However, there are no reports about LTBI treatment results including optimal drug regimen for NKR, even though rifampicin containing regimen supposed to be potent as well considering its efficacy and drug compli- ance $^{12,13}$.

The purpose of this study was to compare the status of LTBI treatment between NKR and South Korean Contacts (SKC), and to investigate factors affecting drug compliance for LTBI treatment in NKR.

\section{Materials and Methods}

\section{Study population}

Limited LTBI screening data of NKR in 2008 when LTBI treatment program was consulted by Korean Institute of $\mathrm{Tu}$ berculosis were selected. As comparative data, data of TB contacts investigated in closed correctional facility of SK where consecutive active TB developed in 2007 were reviewed $^{14,15}$. In

Table 1. Clinical characteristics of tuberculin skin test reactors $(\geq 10 \mathrm{~mm})$ who underwent latent tuberculosis screening

\begin{tabular}{|lccc|}
\hline & SKC (n=126) & NKR (n=172) & p-value \\
\hline Male sex & $91(72.2)$ & $34(19.8)$ & $<0.01$ \\
\hline Age, yr & $42.8 \pm 9.9$ & $35.4 \pm 10.0$ & $<0.01$ \\
\hline BCG scar & $84 / 126(66.7)$ & $122 / 166(73.5)^{*}$ & 0.21 \\
\hline TB close contact history & $126(100)^{\dagger}$ & $166(96.5)^{\dagger}$ & 0.04 \\
\hline BMI, kg/m ${ }^{\dagger}$ & $22.1 \pm 3.0$ & $22.3 \pm 2.4$ & 0.76 \\
\hline Comorbidity & $6(4.8)$ & $10(5.8)$ & 0.89 \\
\hline Cardiovascular disease & $3(2.4)$ & $7(4.1)$ & \\
\hline Asthma & $0(0)$ & $3(1.7)$ & 0.03 \\
\hline Diabetes mellitus & $3(2.4)$ & $0(0)$ & $<0.01$ \\
\hline TST induration size, mm & $16.6 \pm 4.2$ & $17.4 \pm 3.9$ & $<0.01$ \\
\hline QFT-GIT positive & $101 / 123(82.1)$ & $103 / 103(100)$ & \\
\hline LTBI treatment initiation & $88(69.8)$ & $90(52.3)$ & 0.28 \\
\hline 4R & $88(100)$ & $82(47.7)$ & 0.33 \\
\hline 9H & $0(0)$ & $40(22.3)$ & 0.03 \\
\hline Adverse drug reaction & $22(17.5)$ & $17(9.9)$ & 0.85 \\
\hline Gastrointestinal & $18(14.3)$ & $14(8.1)$ & 0.26 \\
\hline Flu like symptom & $2(1.6)$ & $3(1.7)$ & 0.01 \\
\hline Dermatological & $1(0.8)$ & $6(3.5)$ & \\
\hline Others & $1(0.8)$ & $79 / 90(87.8)$ & $38 / 82(46.3)$ \\
\hline LTBI treatment completion & $77 / 88(87.5)$ & & \\
\hline 4R & $77 / 88(87.5)$ & NA & \\
\hline 9H & $117 / 172(68.0)$ & \\
\hline
\end{tabular}

Values are presented as number (\%), mean \pm standard deviation, or $\mathrm{n} / \mathrm{N}(\%)$.

*BCG scars were examined in 166 subjects among 172 NKR. ${ }^{+} \mathrm{TB}$ close contact history to an index case existed in SKC in closed correctional facility. ${ }^{*}$ TB close contact history to a member of family existed in NKR.

SKC: South Koreans Contacts; NKR: North Koreans Refugees; BCG: bacillus Calmette-Guérin; TB: tuberculosis; BMI: body mass index; TST: tuberculin skin test; QFT-GIT: QuantiFERON-TB Gold In Tube; LTBI: latent tuberculosis infection; 4R: 4 months of treatment with rifampicin; 9H: 9 months of treatment with isoniazid; NA: not applicable. 
the NKR group, in perspective of LTBI treatment, data of TST positive reactors were only available. TST negative results (TST $<10 \mathrm{~mm}$ ) in SKC group were excluded for comparison. As a result, a total of 126 SK facility inmates and 172 NKR were retrospectively reviewed and compared. Written informed consent was obtained, and this study was approved by the Institutional Review Board (IRB) of Korean Institute of Tuberculosis (IRB number: KIT-2007-01/2009-04).

\section{Policy of TB and LTBI screening for NKR}

Basic health screenings including TB and LTBI are initially conducted in National Intelligence Service of SK after immigration. Although TST and confirmatory QFT-GIT had been used (two step strategy) for LTBI diagnosis in the past, recently single QFT-GIT method is being used after excluding active TB using chest radiograph (CXR) and sputum acid fast bacilli smear and culture. Initiated LTBI treatment or TB treatment for 1-2 months is continued at Hana-medical office in settlement support center (Hanawon) for NKR where 3 months' detention and education program is given. Their overlong further medical treatments are referred to near assigned hospital after their new home settlement ${ }^{7}$.

\section{LTBI diagnosis}

TST and additional QFT-GIT (Cellestis, Ltd, Carnegie, Australia) tests were performed as a screening test for LTBI after exclusion of active TB based on CXR in SKC and NKR groups, respectively. A TST was performed using the Mantoux method (Statens Serum Institute [SSI], Copenhagen, Denmark). The induration size was measured after $48-72$ hours by experienced nurses. An induration size with TST $\geq 10 \mathrm{~mm}$ was set as cut-off value. Additional QFT-GIT tests were performed for TST positive reactors while measuring the induration size of

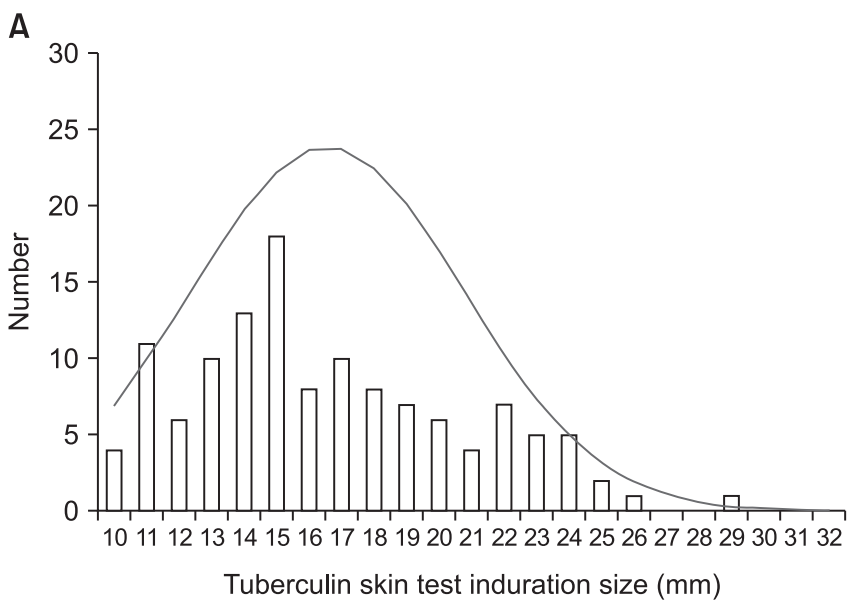

TST. Interpretation of QFT-GIT tests followed the manufacturer's instructions ${ }^{16}$.

\section{LTBI treatment}

LTBI treatment was recommended for subjects with positive results of additional confirmative QFT-GIT results among TST positive reactors in both groups. But some subjects received LTBI treatment irrespective of QFT-GIT results, if they wanted to receive LTBI treatment. As LTBI treatment regimens, 4 months of rifampicin (4R) was used in SKC and 9 months of isoniazid $(9 \mathrm{H})$ or $4 \mathrm{R}$ were prescribed to individuals of the NKR after consultations to a charge doctor in Korean Institute of Tuberculosis. LTBI treatment and adverse drug reaction was monitored for both groups. LTBI treatment completion was defined as finishing $\geq 80 \%$ of the prescribed pills.

\section{Statistical analysis}

All analyses were performed using SPSS software version 20.0 (IBM Corp., Armonk, NY, USA). Chi-square test and Mann-Whitney test after checking normal distribution of data were used. All tests for significance were two-sided. Statistical significance was set at $\mathrm{p}<0.05$. Associations of LTBI treatment completion with group, drug regimen, adverse drug reaction, sex, and age were analyzed by binary logistic regression.

\section{Results}

\section{Clinical characteristics}

A total of 126 SK facility inmates who were investigated after TB outbreaks and 172 NKR who underwent immigrants TB screening were enrolled for this study. These characteris-

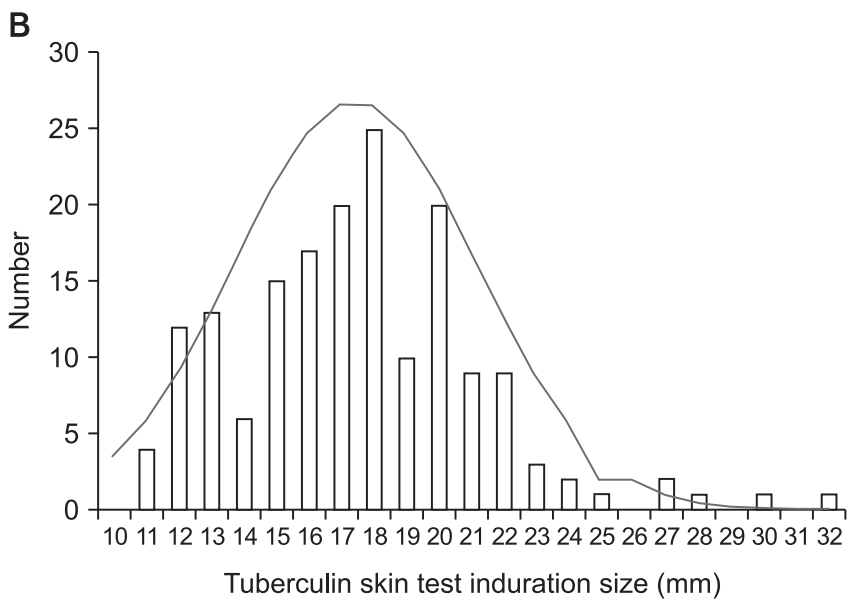

Figure 1. The distribution of tuberculosis skin test positive reactors (induration $\geq 10 \mathrm{~mm}$ ). (A) South Korean contacts. (B) North Korean refugees. 
tics are summarized in Table 1. The ratio of male was higher in SKC $(\mathrm{p}<0.01)$. Mean age was higher in SKC than in NKR $(p<0.01)$. BCG scar rate was not significantly different between the two groups $(\mathrm{p}>0.05)$. TB close contact histories from families existed in NKR (96.5\%) whereas SKC had contact histories to an index infectious TB case (100\%). Comorbidity conditions were not significantly different between the two groups $(p>0.05)$. Mean body mass index was not significantly different between the two groups either $(\mathrm{p}>0.05)$ (Table 1$)$.

\section{Diagnosis and treatment of LTBI}

As shown in Figure 1, the distribution of TST positive reactors were right shifted in NKR compared with SKC. The induration size of TST was $16.6 \pm 4.2 \mathrm{~mm}$ in SKC (A) and $17.4 \pm 3.9 \mathrm{~mm}$ in NKR (B) with significant difference $(\mathrm{p}=0.03)$. The QFT-GIT positive rate was $82.1 \%$ in SKC and $100 \%$ in NKR. LTBI treatment in SKC was initiated for 88 patients (69.8\%), all of whom were prescribed 4R. In NKR, 172 (100\%) people received LTBI treatment initially, of which $52.3 \%$ were prescribed $4 \mathrm{R}$ while the remaining $47.7 \%$ were prescribed 9H. LTBI treatment completion rate was significantly higher in SKC $(77 / 88,87.5 \%)$ than that in NKR $(117 / 172,68.0 \%)$. Among NKR, LTBI completion rate was $87.8 \%$ in $4 \mathrm{R}$ and $46.3 \%$ in $9 \mathrm{H}$, respectively. However, the overall frequency of adverse drug reaction was not different between two groups (Table 1). During 2 years of follow-up after LTBI treatment, one active TB developed in SKC, but none in NKR.

\section{Factors associated with LTBI treatment completion}

When LTBI treatment completion rate was analyzed in NKR as a binary logistic regression, $4 \mathrm{R}$ regimen (odds ratio [OR], 9.30; 95\% confidence interval [CI], 4.16-20.77; $<<0.01$ )

Table 2. Binary logistic regression with adjustment for latent tuberculosis infection treatment completion in $\mathbf{1 7 2}$ NKR

\begin{tabular}{|lccc|}
\hline & \multicolumn{3}{c|}{ NKR } \\
\cline { 2 - 4 } & Odds ratio & $\mathbf{9 5 \%}$ CI & p-value \\
\hline $4 \mathrm{R}$ & 9.30 & $4.16-20.77$ & $<0.01$ \\
$9 \mathrm{H}$ & Reference & & \\
$\mathrm{ADR}(-)$ & 1.82 & $0.77-4.31$ & 0.18 \\
$\mathrm{ADR}(+)$ & Reference & & \\
Male & 3.45 & $1.19-9.97$ & 0.02 \\
Female & Reference & & \\
Age & 1.03 & $1.00-1.07$ & 0.08 \\
\hline
\end{tabular}

NKR: North Korea refugees; CI: confidence interval; 4R: 4 months of treatment with rifampicin; $9 \mathrm{H}$ : 9 months of treatment with isoniazid; ADR: adverse drug reaction. and male sex (OR, 3.45; 95\% CI, 1.19-9.97; $\mathrm{p}=0.02$ ) significantly contributed to the increased LTBI completion rate in NKR after adjusting for other variables, although age showed borderline statistical significance (OR, 1.03; 95\% CI, 1.00-1.07; $\mathrm{p}=0.08$ ) (Table 2).

\section{Discussion}

As a LTBI treatment regimen for NKR, 4R regimen with shorter period showed higher completion rate than $9 \mathrm{H}$. However, larger study including other regimen with long-term follow-up must be conducted for NKR.

As shown in Figure 1, distribution of TST for positive results in NKR was shifted to the right than that in SKC where older ages were distributed. Although NKR were not proven TB close contacts in a TB outbreak setting as in SKC where exposure time to an active TB index case was very long in a closed correctional facility, casual TB contacts in NKR were supposed to have been frequent and extensive in ordinary life ${ }^{17}$. In addition, considering high QFT-GIT positive rate among TST reactors (TST $\geq 10 \mathrm{~mm})(100 \%, 103 / 103)$, further study about the role of additional QFT-GIT in relation with TST size must be elucidated.

The initiation rate for LTBI treatment was much higher in NKR than that in SKC. This was because NKR were protected and managed by a governmental isolated secret facility (Hanawon) medically and politically in the initial settlement period $^{18}$. However, the overall rate of LTBI treatment completion in NKR was not high because LTBI completion had been dependent on sex and drug regimen, not on adverse drug reactions. Low completion rate in $9 \mathrm{H}$ can be originated from the lesser attention after home settlement out of Hanawon.

Based on our results, special attention is needed for female NKR on LTBI treatment completion, and shorter regimen including rifampicin regimen such as $4 \mathrm{R}$ or $3 \mathrm{HR}$ should be recommend for NKR for better compliacne ${ }^{19,20}$, considering isoniazid abuse in the underground market, "Jangmadang" in $\mathrm{NK}^{21}$. And if possible, Directly Observed Treatment, in short course in Hanawon should be performed to increase compliance.

This study has several limitations. First, data of TB contacts with <TST $10 \mathrm{~mm}$ could not be collected or analyzed because access to data of NKR was limited. Second, TB exposure setting was not well defined for NKR, and exposure time to family active TB cases for NKR could not be identified. Lastly, the enrolled number was too small to represent all people of NKR. In conclusion, for NKR, 4R regiment instead of $9 \mathrm{H}$ should be considered as a LTBI treatment regimen. Further larger study about LTBI for NKR should be conducted with easy access to related data for NKR. 


\section{Authors' Contributions}

Conceptualization: Kim HJ (for Hee Jin Kim), Lee SH. Methodology: Cha JH, Kim HJ (for Hee Jin Kim), Lee SH. Formal analysis: Kim BK, Cha JH, Kim CY, Kim Y, Lee SH. Investigation: Kim HJ (for Hee Jin Kim), Kim HJ (for Ho Jin Kim), Lee JB, Jeon J, Lee SH. Writting - original draft preparation: Kim BK, Kim HJ (for Ho Jin Kim), Kim HJ (for Hee Jin Kim), Lee SH. Writing - review and editing: Kim BK, Kim HJ (for Hee Jin Kim), Kim Y, Kim JH, Shin C, Lee SH. Approval of final manuscript: all authors.

\section{Conflicts of Interest}

No potential conflict of interest relevant to this article was reported.

\section{Funding}

No funding to declare.

\section{References}

1. Korea Centers for Disease Control and Prevention. Annual report on the notified tuberculosis in Korea, 2018 [Internet]. Cheongju: Korea Centers for Disease Control and Prevention; 2019 [cited 2019 Apr 30]. Available from: http://tbzero.cdc. go.kr/tbzero/board/boardView.do?leftMenuId=48\&paramM enuId=77\&boardSeq=5464\& crudType=R.

2. World Health Organization. Implementing the end TB strategy: the essentials. WHO/HTM/TB [Internet]. Geneva: World Health Organization; 2015 [cited 2019 Apr 30]. Available from: https://www.who.int/tb/publications/2015/The_Essentials_ to_End_TB/en/.

3. Cho KS. Tuberculosis control in the Republic of Korea. Epidemiol Health 2018;40:e2018036.

4. Min GH, Kim Y, Lee JS, Oh JY, Hur GY, Lee YS, et al. Social and clinical characteristics of immigrants with tuberculosis in South Korea. Yonsei Med J 2017;58:592-7.

5. Lee S, Ryu JY, Kim DH. Pre-immigration screening for tuberculosis in South Korea: a comparison of smear- and culturebased protocols. Tuberc Respir Dis 2019;82:151-7.

6. Choi CM, June JH, Kang CI, Park JT, Oh SY, Lee JB, et al. Tuberculosis among dislocated North Koreans entering Republic of Korea since 1999. J Korean Med Sci 2007;22:963-7.

7. Ann SY, Ryou SH, Kim SB. Clinical characteristics of defectors from North Korea visiting a single tertiary hospital in South Korea. Korean J Med 2015;89:54-63.
8. Farhat M, Greenaway C, Pai M, Menzies D. False-positive tuberculin skin tests: what is the absolute effect of BCG and non-tuberculous mycobacteria? Int J Tuberc Lung Dis 2006; 10:1192-204.

9. Joint Committee for the Revision of Korean Guidelines for Tuberculosis; Korean Centers for Disease Control and Prevention. Korean guidelines for tuberculosis. 3rd ed. Cheonju: Korea Centers for Disease Control and Prevention; 2017.

10. Fox GJ, Dobler CC, Marais BJ, Denholm JT. Preventive therapy for latent tuberculosis infection-the promise and the challenges. Int J Infect Dis 2017;56:68-76.

11. Chen S, Zhang H, Pan Y, Long Q, Xiang L, Yao L, et al. Are free anti-tuberculosis drugs enough? An empirical study from three cities in China. Infect Dis Poverty 2015;4:47.

12. Venkatesan P. Changing the treatment landscape for latent tuberculosis with rifampicin. Lancet Respir Med 2018;6:740.

13. Menzies D, Adjobimey M, Ruslami R, Trajman A, Sow O, Kim $\mathrm{H}$, et al. Four months of rifampin or nine months of isoniazid for latent tuberculosis in adults. N Engl J Med 2018;379:44053.

14. Lee SH, Lew WJ, Kim HJ, Lee HK, Lee YM, Cho CH, et al. Serial interferon-gamma release assays after rifampicin prophylaxis in a tuberculosis outbreak. Respir Med 2010;104:448-53.

15. Lee SH, Yim JJ, Kim HJ, Shim TS, Seo HS, Cho YS, et al. Adverse events and development of tuberculosis after 4 months of rifampicin prophylaxis in a tuberculosis outbreak. Epidemiol Infect 2012;140:1028-35.

16. Diel R, Loddenkemper R, Meywald-Walter K, Gottschalk R, Nienhaus A. Comparative performance of tuberculin skin test, QuantiFERON-TB-Gold In Tube assay, and T-Spot.TB test in contact investigations for tuberculosis. Chest 2009;135: 1010-8.

17. Seung KJ, Linton SW. The growing problem of multidrugresistant tuberculosis in North Korea. PLoS Med 2013;10: e1001486.

18. Kim YN. Narrative Inquiry about entering process South Korea, NIS investigation course and Hanawon center curriculum of North Korean defectors. J Hum Rights Law-Relat Educ Res 2016;9:33-64.

19. Park SJ, Jo KW, Yoo B, Lee CK, Kim YG, Yang SK, et al. Comparison of LTBI treatment regimens for patients receiving anti-tumour necrosis factor therapy. Int J Tuberc Lung Dis 2015;19:342-8.

20. Assefa Y, Assefa Y, Woldeyohannes S, Hamada Y, Getahun H. 3-month daily rifampicin and isoniazid compared to 6- or 9-month isoniazid for treating latent tuberculosis infection in children and adolescents less than 15 years of age: an updated systematic review. Eur Respir J 2018;52:1800395.

21. Park SM, Lee HW. Current status of healthcare and effective health aid strategies in North Korea. J Korean Med Assoc 2013;56:368-74. 